\title{
How the Genome Folds, Divides, Lives, and Dies
}

\author{
Whitney L. Johnson, ${ }^{1,2}$ Kathleen T. Xie, ${ }^{1,2}$ Mijung Kwon, ${ }^{1,2,5}$ Shiwei Liu, ${ }^{1,2,5}$ \\ and David Pellman ${ }^{1,2,3,4}$ \\ ${ }^{1}$ Department of Pediatric Oncology, Dana-Farber Cancer Institute, Boston, Massachusetts 02215 \\ ${ }^{2}$ Department of Cell Biology, Harvard Medical School, Boston, Massachusetts 02215 \\ ${ }^{3}$ Broad Institute of MIT and Harvard, Cambridge, Massachusetts 02142 \\ ${ }^{4}$ Howard Hughes Medical Institute, Chevy Chase, Maryland 20815 \\ Correspondence: david_pellman@dfci.harvard.edu
}

The 82nd Cold Spring Harbor Symposium was the first to focus on Chromosome Segregation and Structure. In one sense, this was something new. There has been significant recent progress on chromosome segregation and chromosome structure, and it was a propitious time to bring these fields together. But in another sense, the topics discussed at the meeting were classic ones that have been central to many prior Cold Spring Harbor Symposia, going back decades. In looking back over prior meetings, it is stunning to see the progress that has been made in understanding genes, chromatin, cell division, cancer, and chromosome biology in general. Remarkably, about 80 years ago, it was still valid to ask whether chromosomes are even worth studying. Summing up the 1941 meeting, H.J. Muller laconically noted, "The time has come when a few ... of the physicists and biochemists realize that genes and chromosomes do form valid subjects of inquiry, and that what the biologists have been saying about their mysterious properties calls for some more looking into on their part." Fortunately, long before the current symposium, Muller's view prevailed, and the debate was settled on whether chromosomes are indeed a valid subject for inquiry. Even so, for many years, chromosome biology research was limited by the available tools. In summarizing the 1973 meeting, Hewson Swift said, with some frustration, that eukaryotic chromosomes under the electron microscope looked, “.... even at their best, something like a bad day at a macaroni factory." (Pasta has long been a favored metaphor for chromosomes.) Nowadays, we can make a bit more sense from our observations of genomic noodles. For example, at the current meeting, we learned that the "vermicelli"like appearance of WAPL-depleted chromatin can provide deep insights into the organizing principles of chromosomes. New technologies have been applied to classic problems and have pushed the field forward in exciting directions. So, by 2017, we had a better day at the "macaroni factory."

The 82nd Symposium was a concentrated five-day burst of information. In addition to highlighting a cross section of the most exciting cutting-edge work, the meeting was broad and provided a crash course on relevant topics for scientists looking to bridge fields. The organizers-Terri Grodzicker, David Stewart, and Bruce Stillman-put together an impressive program with an exciting list of speakers. An interesting side note: Cold Spring Harbor Symposia used to be more of a way of life than a focused event. In the 1941 meeting summary, it was noted that Milislav Demerec made a major organizational shift by shortening the meeting from the customary five weeks to two! Wistfulness over a bygone era of leisurely science aside, the 82nd Symposium illustrated what can be accomplished in five days.

Why have a meeting focused on chromosome segregation and structure now? Many important long-standing questions are being addressed - from the epigenetic specification of centromeres to the requirement of mitotic chromosome compaction for proper segregation. However, one new theme stood out: Taking what we have learned about self-assembly from the spindle and applying it to chromosome structure. Many aspects of spindle assembly are driven by molecular motor proteins that can sort and orient microtubules, measure their lengths, and create intermediate structures such as asters or antiparallel microtubule arrays. Motors can also control signaling within the spindle by concentrating signaling molecules and generating signal gradients. New methods are elucidating these self-assembly design principles, including new live cell imaging methods, structural studies, biochemical reconstitution, and single-molecule imaging. The 82nd Cold Spring Harbor Symposium occurred at an inflection point for the chromosome structure field where, for the first time, a similar understanding of chromosome self-assembly seems within grasp. Here again, motor proteins organize structure, folding the chromatin polymers into intermediate structures using simple biophysical principles and basic biochemical activities. Below, we summarize self-assembly and other major themes that emerged during the meeting, while hoping to still convey the beauty of many individual stories.

\footnotetext{
${ }^{5}$ These authors contributed equally to this work.

(C) 2017 Johnson et al. This article is distributed under the terms of the Creative Commons Attribution-NonCommercial License, which permits reuse and redistribution, except for commercial purposes, provided that the original author and source are credited.
} 


\section{SELF-ASSEMBLY AND REGULATION OF THE CHROMOSOME SEGREGATION MACHINERY}

The symposium had numerous talks highlighting progress on understanding the mitotic spindle and its coordination with chromosomes, nuclear envelopes, and cytokinesis.

\section{How to Build a Spindle and Know if You Got It Right}

Tubulin molecules assemble into dynamic microtubule polymers, which must organize into higher-order spindle structures - a classic nonequilibrium self-assembly process. Tarun Kapoor reviewed work from his laboratory demonstrating that a microtubule motor (kinesin-4) and a nonmotor microtubule-associated protein (PRC1) can serve as measuring devices, by accumulating at microtubule ends in a cluster whose size is proportional to the length of the microtubule (Subramanian et al. 2013). Using light sheet microscopy, his group is studying how these tagged microtubules self-assemble into a functioning spindle midzone capable of driving anaphase B spindle elongation. He also described the viscoelastic material properties of the spindle. For fast-acting forces, the spindle behaves as an elastic solid that adapts to applied force. For slow-acting forces comparable to the movement of chromosomes, the spindle is more fluid, allowing chromosomes to pass through while maintaining spindle integrity.

Microtubules assemble into spindles, but they must also interact with chromosomes to facilitate chromosome segregation. Stephen Harrison addressed the design principles of the kinetochore, the site of attachment of the chromosome to the mitotic spindle (Jenni et al., this volume). He presented structures of several kinetochore subcomplexes. The subcomplexes are often assembled into higher-order structures via "peptide-in-groove" contacts, where an unstructured peptide of one subcomplex component docks into a structured binding site of another. This type of connection has many benefits: It is amenable to regulation because the peptide is easily accessed by kinases and phosphatases, and the contact also provides mechanical compliance and structural adaptability.

In addition to linking the chromosome and spindle, the kinetochore is also regulatory. Kinetochores that have not yet properly attached to microtubules send a "wait anaphase" signal via the spindle assembly checkpoint (SAC), providing time to correct segregation errors. The SAC prevents mitotic exit by inhibiting the APC/C E3 ligase that degrades mitotic cyclins. Through biochemical and structural studies, there has been tremendous recent progress in understanding the SAC. However, talks at the meeting illustrated that there are also still surprises. Arshad Desai showed that not only can the kinetochore inhibit the APC/C and put the brakes on anaphase entry, but that it can also activate the $\mathrm{APC} / \mathrm{C}$ and accelerate anaphase progression (Lara-Gonzalez et al., this volume). Both of these functions are mediated by $\mathrm{Cdc} 20$, a key regulator of the $\mathrm{APC} / \mathrm{C}$. At unattached kinetochores,
Cdc20 is loaded onto the mitotic checkpoint complex, which inhibits the APC/C. However, Cdc20 dynamically associates with the kinetochore even when microtubules are properly attached. Desai showed that while fluxing through the attached kinetochore, Cdc20 is dephosphorylated and activated, which accelerates mitotic exit. Therefore, it is kinetochore-microtubule attachment that flips the kinetochore from an APC/C "brake" to an APC/C "activator." Jennifer DeLuca also discussed how kinetochores modulate the strength of kinetochore-microtubule attachments during mitosis. Early in mitosis, dynamic attachments are beneficial, so that errors can be readily corrected. Later, stable attachments are beneficial, so that correctly attached chromosomes do not detach from the spindle. It is well known that the mitotic kinase Aurora B facilitates the early lability of attachment through inhibitory phosphorylation of kinetochore proteins. DeLuca presented surprising findings that its cousin Aurora A is also important in this process, although Aurora A is famous for its functions at the centrosome, not the kinetochore (DeLuca, this volume). Thus, although the localization of the majority population of a protein can provide clues about function, minor populations can be important, and should not be overlooked. At anaphase onset, the cohesin remaining between sister chromatids is released in an APC/C-dependent manner. Yoshinori Watanabe found that the conserved meiotic kinetochore protein Moa1/Meikin, although initially identified as a factor that facilitates monoorientation in meiosis I, also protects pericentric cohesin from removal until anaphase II (Miyazaki et al., this volume). Moa1-mediates cohesin protection by increasing both the localization and the activity of Shugoshin (Sgo1), a known cohesin protector. This work uncovers a new protector of meiotic cohesin and shows how one protein can regulate multiple meioticspecific functions.

\section{Three Ways of Looking at a Centrosome}

There were new insights into the structure and assembly of the centrosome. In most animal cells, centrosomes organize the poles of the mitotic spindle. Centrosomes are typically composed of microtubule-containing centrioles surrounded by an amorphous protein mass called the pericentriolar material (PCM). Using recombinant $C$. elegans proteins, Tony Hyman's group showed that even in the absence of microtubule-containing centrioles, PCM can spontaneously self-assemble and nucleate microtubules. The scaffold protein SPD-5 undergoes a phase transition, forming liquid-like droplets, which can sequentially recruit other PCM proteins and concentrate enough unpolymerized tubulin to nucleate microtubule asters. Tim Stearns showed that centriole triplet microtubules are required to maintain the structural integrity of centrioles (Wang and Stearns, this volume). Human cells that lack $\delta$-tubulin or $\varepsilon$-tubulin form defective centrioles without normal triplet microtubules, fail to undergo centriole maturation, and disintegrate during mitotic exit by an interesting but still mysterious process. Mónica Bettencourt-Dias addressed 
how centrioles are eliminated in female meiosis so that the zygote has a normal centriole number after fertilization. Her work demonstrates that centrosome reduction starts with transcriptional repression of Polo kinase during oogenesis (Nabais et al., this volume). Loss of Polo leads to the loss of PCM, which in turn is required to maintain centrioles. The reputation of the PCM has suffered somewhat by association with the adjective "amorphous." The talks described above provided a rehabilitation of sorts, highlighting PCM's central role in centrosome integrity and in development.

\section{The Spindle Is the Center of the Universe?}

Spindle function has to be coordinated with many other cellular processes, which was the topic of several talks. Tim Mitchison described how, in large frog eggs, microtubules asters from each spindle pole grow all the way to the cell cortex, and their region of overlap defines the site of cytokinetic furrow formation (Mitchison and Field, this volume). Interestingly, in polyspermic eggs, the cleavage furrow only forms between asters from the same spindle, with chromatin in between them. Here, again, motors are key. Motors transport the chromosome passenger complex (CPC) from chromosomes to the aster overlap zone, and only overlapping asters with the CPC define the site of the cleavage furrow. Thus, chromosomes can direct cytokinetic furrow positioning, even at large distances in huge egg cells. As meeting participant Bill Earnshaw has previously noted (Earnshaw and Bernat 1991), these experiments illustrate that Daniel Mazia, although witty, got it wrong when he said, "Indeed, the role in mitosis of the chromosome arms, which carry most of the genetic material, may be compared with that of a corpse at a funeral: they provide the reason for the proceedings but do not take an active part in them" (Mazia 1961).

At the end of chromosome segregation in metazoan cells, the nuclear envelope (NE) reforms around the daughter chromosomes masses, and all of the chromosomes must be encapsulated to form a single nucleus. Daniel Gerlich used an imaging-based RNAi screen to identify mutants that form multiple nuclei. The screen revealed that loss of BAF (barrier-to-autointegration factor) resulted in the formation of micronuclei, which are miniature additional nuclei that contain one or a few chromosomes and are commonly observed in cancer. A satisfying combination of imaging and biophysical experiments showed that BAF forms a stiff shell around decondensing chromosomes, thus keeping them together as one mass. Interestingly, the mechanical properties of BAF can be regulated by phosphorylation, which ensures that the stiff shell persists for only a short period, until the NE reforms. Our laboratory (David Pellman's laboratory) addressed the coordination between chromosome segregation and NE assembly, which is relevant to understanding the properties of micronuclei. We had previously shown that chromosomes encapsulated in micronuclei are prone to DNA damage and chromothripsis, a type of large-scale genome rearrangement. Spontaneous disruption of the micronu- clear envelope triggers DNA damage (Hatch et al. 2013). We found that this fragility of micronuclei can be explained by a defect in NE assembly. Chromosomes that separate from the main chromosome mass lag within the spindle, where spindle microtubules occlude key NE components. The data suggest that rather than the tight, checkpoint-mediated coordination between chromosome segregation and $\mathrm{NE}$ assembly that was previously proposed, there is only loose coordination from the normal timing of spindle disassembly. The absence of precise regulatory controls may explain why errors during mitotic exit are frequent and a major source of catastrophic genome rearrangement.

Recently, it has been shown that NE disruption (e.g., in micronuclei) can activate an innate proinflammatory response. This response occurs because cytoplasmic DNA, which the cell interprets to be foreign DNA, activates the cytoplasmic DNA sensor cGAS (cyclic GMP-AMP synthase). However, in mitosis, genomic DNA is also exposed to the cytoplasm and yet does not trigger innate immune signaling. Using both Xenopus extracts and cultured mammalian cells, Hiro Funabiki showed that while mitotic chromatin can bind cGAS, the presence of nucleosomes inhibits cGAS activation, providing a crude first discrimination between self (chromatinized) DNA and foreign (naked) DNA (Funabiki et al., this volume). However, in the presence of drugs that prolong mitosis, cGAS does eventually become activated, promoting apoptosis.

\section{Life after HeLa Cells}

We have learned a tremendous amount about cell division and chromosome biology from tissue culture cells and single cell model organisms. However, participants at the meeting also sought to understand the diversity of cell functions within normal tissue and development. Multiple speakers addressed how cell extrinsic factorssuch as in vivo tissue architecture and cell-cell contactsimpact chromosome segregation and cell division. Angelika Amon showed that complex three-dimensional (3D) tissue architecture promotes accurate chromosome segregation and genome stability. She reported that the frequency of chromosome missegregation is higher in 2D tissue cultures than in 3D organoid cultures or in tissues. Cell nonautonomous mechanisms, such as cell-cell contacts and cell-matrix adhesion, are known to influence the fidelity of chromosome segregation. Dissecting other features of the normal 3D tissue microenvironment that enhance segregation fidelity remains a fascinating problem. Eric Nigg reported new effects of the centrosome on tissue architecture (Nigg et al., this volume). He found that inducing centrosome structural aberrations (overexpression of NLP) disrupted 3D spheroid structure. This disruption occurs because cells with centrosome aberrations are stiff and squeeze dividing cells out of the epithelium, affecting even those cells without NLP overexpression. This study provides an interesting physical example of a cell nonautonomous effect. Finally, Jan van Deursen addressed the 100-year-old hypothesis from Theodor Boveri that centrosome abnormalities can cause aneuploidy and promote 
tumorigenesis. He reviewed the many mouse models that have been generated to test the hypothesis and highlighted that we still do not understand why some models generate tumors and others do not. He proposed that defects in the separation of centrosomes, which cause abnormal spindle geometry, could be an important feature of the more tumorigenic models.

\section{SELF-ASSEMBLY AND REGULATION OF CHROMOSOME STRUCTURE}

The 82nd Cold Spring Harbor Symposium marked and celebrated a major step forward in the field of chromosome structure. The causes and consequences of chromosome structure have been a central mystery in biology since the discovery of the mitotic chromosome cycle by Walther Flemming and the subsequent observation that the interphase nucleus is partitioned into euchromatin and heterochromatin by Emil Heitz. Now, a quantitative and testable model - termed the loop extrusion modelappears to explain key aspects of chromosome structure as a self-organization process driven by motor proteins. It is proposed that the dramatic difference in the appearance of mitotic and interphase chromosomes may be something of an illusion. Instead of differing by mechanistically distinct organizing principles, mitotic and interphase chromosomes may instead represent a continuum in the degree of looped chromosome organization. A host of new technologies have driven these breakthroughs.

\section{The Basics of Loop Extrusion}

Leonid Mirny described the loop extrusion model and summarized its history, starting with earlier versions by Arthur Riggs, John Marko, and meeting participant Kim Nasmyth (Riggs 1990; Nasmyth 2001; Alipour and Marko 2012). The core idea of the model is that chromosomes become organized into loops, by motor proteins ("loop extruders") that pump DNA processively to generate loops (Fudenberg et al., this volume). These loop extruders can accumulate in certain regions of the genome if they encounter a barrier, either by colliding into each other, or the transcription regulator CTCF. Because CTCF binding is sequence-dependent, this particular barrier creates a pattern of loops that is similar between individual cells and is therefore detectable by population-average experiments. These semistable loops form insulated regions termed topologically associated domains (TADs) that have been detected in chromosome conformation capture experiments (mostly $\mathrm{Hi}-\mathrm{C}$ ).

The function of the molecules driving loop extrusion are rapidly being defined. The major, if not only, loop extruders are the SMC complexes (structural maintenance of chromosomes), including cohesin and condensin. These complexes share an overall similar ring-shaped structure, with two proteins (each forming long antiparallel coiled coils) being joined at a "hinge" on one end, and two ATPase "head" domains at the other end. The heads can dimerize in the presence of ATP and are additionally linked by kleisin proteins, forming a double gate. As discussed by Mirny, the current loop extrusion model makes quantitative and testable predictions about the consequences of eliminating loop extruders, increasing their density or processivity, and eliminating extrusion barriers. Indeed, other talks at the symposium and work published after the meeting validate these predictions. SMC-dependent loop extrusion has now been observed by live-cell imaging in Bacillus subtilis (Wang et al. 2017), and single molecule imaging experiments show that SMC proteins are processive DNA motors capable of loop extrusion in vitro (Terakawa et al. 2017; Ganji et al. 2018).

Jan-Michael Peters also presented work validating the loop extrusion model, showing that CTCF is required for TAD patterning in interphase. Additionally, his group observed that if cohesin cannot be removed from chromosomes (by depletion of WAPL, a protein required for cohesin removal), chromosomes condense into structures that visually resemble prophase chromosomes; these prophase-like structures are termed "vermicelli" (Tedeschi et al. 2013). Hi-C analysis revealed that WAPL-depleted chromosomes show extensive looping which can even extend beyond CTCF boundaries (presumably due to the increased lifetime of cohesin on chromatin after its removal factor has been depleted) (Haarhuis et al. 2017; Wutz et al. 2017). Interestingly, Hi-C analysis in mitotic cells also revealed extensive looping with boundaries not determined by CTCF at all (boundaries may instead primarily be demarcated by the accumulation of loop extruders colliding with each other) (Gibcus et al. 2018). WAPL-depleted interphase chromosomes thus resemble mitotic chromosomes structurally. These findings suggest the simplifying view that interphase and mitotic chromosome organization exist on a continuum, differing mainly through the activity and concentration of loop extruders as well as the integrity of the extrusion barriers. Why cohesins are the main loop extruders in interphase whereas condensins are the primary extruders in mitosis remains to be determined.

What are the minimal requirements to make a mitotic chromosome? Tatsuya Hirano used biochemical reconstitution to show that condensins (I and II) are sufficient to organize DNA into a mitotic chromatid-like structures, even in the absence of histones (Shintomi and Hirano, this volume). Although these nucleosome-depleted structures were sparser and more fragile, linear compaction of mitotic DNA occurs efficiently, presumably via DNA looping. Hirano reminded the participants that condensins serving as the fundamental architects of mitotic chromosome should not be that surprising, given that condensins are conserved in bacteria whereas histones are not.

Kerry Bloom described the improvement of a statistical mechanics model of DNA structure by including loop extruding motors into his simulations of chromatin (Lawrimore et al., this volume). The entropy-driven tendency of floppy chromatin fibers to form loops suggests that loop extruders may be able to make local jumps, which might explain the large step sizes recently observed during loop extrusion (Ganji et al. 2018). Moreover, Bloom noted that local tethers on chromatin can cause an accumulation of loop extruders. Because local tethers occur at centromeres 
due to microtubule attachment, this model may explain the observed pattern of condensin accumulation around centromeres. Thus, Bloom's model suggests ways in which the spindle microtubules can pattern the organization of chromatin.

\section{Put a Ring on It}

SMC proteins can be loaded onto DNA in several ways. If the SMC ring is opened, DNA can enter the ring, and after the ring closes, the SMC encloses the DNA (a topological interaction). Alternatively, DNA can bind the ring directly or enter the ring as a loop, without the SMC ring opening (a nontopological interaction). Talks from Kim Nasmyth and Frank Uhlmann addressed the mechanisms that enable cohesins to toggle between the two different modes of interaction with DNA. Topological interactions are important for sister chromatin cohesion, whereas nontopological interactions likely enable loop extrusion. Supporting this idea, Nasmyth described separation-of-function mutations of cohesin that enable nontopological interactions but abolish topological interactions and sister chromatid cohesion. These results provide definitive evidence for cohesin loading onto DNA in two ways.

For dynamic topological interactions to occur, DNA must be able to enter and exit the cohesin ring. All groups working on the problem agree on the cohesin release mechanism, which either involves kleisin cleavage at anaphase or dissociation of the kleisin-SMC3 interface. However, there is no consensus on the DNA entry mechanism. Protein cross-linking and protein fusion experiments from Nasmyth's laboratory suggest that cohesin is a one-way street, with DNA entry through the hinge and exit from SMC head-kleisin gate. In contrast, Frank Uhlmann's work suggests that the SMC head-kleisin gate is reversible, used for both entry and exit. It is possible that both junctions can allow entry, but which is the dominant path remains to be determined. To obtain functional cohesion, after the DNA from one chromatid loads, the sister chromatid must also be captured. A previous model for sister chromatid capture involved "replication-through-aring," in which the replisome passes through the ring, and thus both daughter strands of DNA are encircled without necessarily requiring ring opening. However, Uhlmann presented appealing biochemical data for an alternative model, where the sister chromatids may be incorporated into the ring in two steps. First, SMC opens to capture double-stranded DNA from the leading strand during replication. Then, SMC opens again to capture singlestranded DNA from the lagging strand.

\section{Organization and Regulation at All Length Scales}

Ideally one would want to understand the principles of chromatin self-organization at all scales from the nucleosome all the way up to a single chromosome territory. Guohong Li described his work on cryo-EM structures of small arrays of nucleosomes that form $30 \mathrm{~nm}$ filaments
(Chen and $\mathrm{Li}$, this volume). The new high-resolution structures discriminate between competing models in the field, establishing that 12 nucleosome arrays form a zigzag twostart nucleosome stack. Recalling Stephen Harrison's talk, knowing the binding junctions that define nucleosome compaction enables biophysical experiments to better define sites of regulation-for example, how histone variants influence compaction. It makes sense that these fundamental biochemical properties of nucleosome arrays in vitro will be relevant in vivo, but this still remains something of a leap of faith, as recent studies have not universally detected 30-nm filaments in vivo (Lieberman-Aiden et al. 2009; Fussner et al. 2012; Nishino et al. 2012). It is possible that short segments of $30 \mathrm{~nm}$ fibers do form in vivo but that current methods fail to detect them. Ollie Rando described Micro-C, a method that enables small scale analysis of the structure of nucleosome arrays in vivo. Micro-C data could be consistent with the formation of short segments of 30-nm chromatin fibers in yeast. Additionally, although yeast do not have typical TADs, with Micro-C Rando's group was able to detect the organization of small numbers of genes into TAD-like "gene crumples."

The functional impact of chromosome organization on gene expression is still a complex and open question. Several talks addressed the functional consequences of chromosome organization and revealed new wrinkles on the interplay between chromatin organization and the regulation of gene expression. Edith Heard showed that during $\mathrm{X}$-chromosome inactivation, the inactive $\mathrm{X}$ chromosome (Xi) is largely devoid of TADs (Galupa and Heard, in this volume). The $\mathrm{Xi}$ is instead organized into two large selfinteracting mega-domains, separated by a region containing the DXZ4 macrosatellite sequence. Like TADs, the formation of such mega-domains is proposed to facilitate the expression of certain genes on $\mathrm{Xi}$ that can escape silencing, as loss of megadomains by DXZ4 deletion prevents facultative gene expression escape on the mouse Xi (Giorgetti et al. 2016). The resulting large-scale restructuring of the chromosome therefore generates changes in gene expression, albeit on a modest scale.

TADs and mega-domains affect gene expression, but Gerd Blobel suggested that a transcriptional regulator can also help form TAD boundaries (Hsu and Blobel, this volume). He showed that a chromatin reader protein of the BET family, BRD2, colocalizes with CTCF genome wide. BRD2 helps form chromatin boundaries, both by limiting the spread of enhancer activity, and by maintaining the structural integrity of domain boundaries. Clinically relevant BET inhibitors may therefore perturb gene expression through disruption of TAD boundaries, not just by direct transcriptional effects. Allan Spradling described the linkage between TAD boundaries and DNA replication timing domains in Dipteran polytene chromosomes (Spradling, this volume). Here, some TAD boundaries are thought to cause replication fork stalling and breakage, leading to the formation of underreplicated regions and a heterogeneous array of deletions within these sequences. Under-replication and the loss of late replicating heterochromatic sequences could provide a genetic basis for gene silencing. 


\section{EPIGENETIC REGULATION OF THE GENOME}

In addition to chromatin loops and TADs, other mechanisms contribute to genome regulation. Specialized chromatin states are established by DNA modifications, histone modifications, or histone variants. Chromatin states can propagate epigenetically, independent of DNA sequence. Talks at the Symposium highlighted important progress in this area, in several cases defining interesting connections to chromosome segregation.

\section{Centromeres and Chromatin States Required for Chromosome Segregation}

A classic example of epigenetic regulation is the centromere, a site on each chromosome where the microtubulebinding kinetochore is built during mitosis. Centromeres in most organisms are thought to be defined by a histone H3 variant, CENP-A, which not only recruits kinetochore proteins but also facilitates its own assembly into chromatin, thus propagating epigenetically. Centromeres are so crucial to proper chromosome segregation that one might expect their composition and organization to be highly conserved. However, Steven Henikoff reminded us of the remarkable diversity of centromere organization across organisms (Henikoff et al., this volume). For example, centromeres in worms and some insects are holocentric (distributed across the entire chromosome), whereas centromeres in other species are monocentric (localized at a single primary constriction). Centromere DNA sequences are also one of the fastest evolving regions of the genome. To explain the rapid evolution of centromeric DNA sequences and CENP-A, Henikoff proposed the "centromere drive hypothesis," which he developed with Harmit Malik (Henikoff et al. 2001; Henikoff and Malik 2002). The centromere drive hypothesis posits that centromeric sequence variants act as selfish DNA elements, competing to be retained in the egg rather than discarded into a polar body during female meiosis. This favors the emergence of "stronger" and sometimes larger centromeres. What prevents these centromeres from taking over the chromosome is a fitness cost during male meiosis, which selects for new CENP-A variants that neutralize the effects of the expanding centromere.

The centromere drive hypothesis is not only a fascinating genetic phenomenon but also a fundamental cell biology problem. The model requires that stronger centromeres exploit spindle asymmetry to enable their preferential segregation to the egg. Ben Black and Michael Lampson reported exciting progress in elucidating the cell biological basis of centromere drive in mouse meiosis I (Lampson and Black, this volume). Previous work showed that RAN gradients around chromosomes establish a concentration of the CDC42 GTPase at the cortex near the spindle. Black and Lampson showed that localized activity of CDC42 establishes asymmetric tubulin modifications within the spindle, and these modified microtubules form less stable attachments to stronger (larger) centromeres/kinetochores. This preferentially ori- ents stronger centromeres toward the egg pole. Although many questions remain, this work initiates a mechanistic understanding of how selfish DNA elements can drive centromere evolution in mice. As discussed by Henikoff, there are likely to be many variations on this theme. He suggested that holocentric chromosome architecture, as is found in nematodes, may have resulted from pressure to escape centromere drive. Moreover, epigenetically defined centromeres may themselves have originated to fight off selfish DNA elements that would otherwise promote their own segregation.

Two other talks also discussed cell division asymmetry and how it may be influenced by centromeres and histone modifications. Xin Chen found that Drosophila male germline stem cells show a biased distribution of histones during their asymmetric cell division (Kahney et al., this volume). After mitosis, newly translated histones end up in the differentiating daughter cell, whereas older histones partition into the daughter stem cell. Chen showed that this asymmetric distribution of histones is possible because of the asymmetry of two distinct processes; first, older histones are preferentially loaded onto the same chromatid during DNA replication, and second, the sister chromatids with older histones are preferentially segregated into the daughter stem cell during mitosis. Importantly, mutation of a specific residue of histone $\mathrm{H} 3$ led to loss of biased chromosome segregation, causing germline stem cell loss and progenitor stem cell tumor phenotypes, and indicating that asymmetric histone inheritance is an important mechanism that determines cell fate. In another talk, Yves Barral provided evidence that the centromere has a global impact on chromosome structure and segregation in the inherently asymmetric cell divisions of budding yeast. He found that without centromeres, chromosomes are unable to recruit Aurora B during mitosis and are therefore unable to phosphorylate histone 3 serine 10 (H3S10) and properly condense chromosomes. Interestingly, artificially tethering Aurora B to acentric chromosomes rescues condensation, and chromosomes become more likely than before to segregate into budding daughter cells.

In addition to recruiting the kinetochore and facilitating chromosome segregation, CENP-A serves as an epigenetic mark by promoting its own local assembly into chromatin. Several talks addressed the determinants for positioning CENP-A on the chromosome. Precise mapping of centromere locations on genomic DNA has been historically difficult due to the highly repetitive nature of centromeric DNA sequences. Steve Henikoff, Don Cleveland, and Ben Black all discussed data on CENP-A positioning in mammalian cells, using de novo clustering of ChIP-seq reads and newly assembled models of centromeric repeats. They found that CENP-A is positioned on centromeric repeats in a precise and stereotypical manner. CENP-A is phased between CENP-B boxes, which are binding sites for CENP-B, the only known sequence-specific centromeric DNA binding protein in humans. Surprisingly, however, Don Cleveland showed that the phasing of CENP-A nucleosomes between CENP-B boxes does not depend on the presence of CENP-B protein. This observation could be explained either by CENP-B being a vestigial 
mechanism for CENP-A positioning that is no longer in use, or by CENP-B impacting CENP-A positioning only over long timescales, after many cell divisions. Steve Henikoff also speculated that CENP-B may impact the local conformation of chromatin to promote CENP-A assembly.

Although CENP-A position appears precise on the chromosome, initial CENP-A loading is not precise. Don Cleveland showed that during CENP-A loading in $G_{1}$, some CENP-A is incorporated incorrectly onto chromosome arms. However, ectopic centromere formation is prevented, because incorrectly placed CENP-A on chromosomes arms is cleared out during $\mathrm{S}$ phase, while correctly placed CENP-A on $\alpha$-satellite repeats remains precisely positioned. Interestingly, Genevieve Almouzni discussed how cancer cells become overly reliant on the system for targeting CENP-A, thus creating a potential therapeutic vulnerability (Sitbon et al., this volume). Both CENP-A and HJURP, a CENP-A assembly chaperone, are overexpressed in cancer cells. If CENP-A is overexpressed, cancer cells become acutely sensitive to HJURP depletion, presumably because HJURP prevents deleterious CENP-A deposition on chromosome arms. Therefore, cancer cells can exhibit an addition to nononcogene, epigenetic factors.

\section{Epigenetic Regulation of Genes and "Junk"}

Epigenetic mechanisms not only ensure maintenance of centromeres but also control transcription. Transcriptional states need to propagate through many cell divisions to maintain cell identity, dosage compensation, and imprinting patterns. Marisa Bartolomei discussed how allelespecific gene expression is disrupted in mouse models of imprinting disorders. She showed that although mutations in imprinting control regions (ICRs) cause the loss of imprinting in primary MEFs, this effect is not homogenous; distinct cell subpopulations show either normal monoallelic or abnormal biallelic expression. These subpopulations show different methylation patterns at their ICRs and never interconvert. This result implies a mechanism where ICR mutant cells make stochastic decisions about ICR methylation status that are stably propagated epigenetically. This mechanism may explain mosaicism seen in human imprinting disorders. Another classic epigenetic phenomenon was discussed by Barbara Meyer, who reported the discovery of a general class of epigenetic regulators from studies of $C$. elegans $\mathrm{X}$ chromosome dosage compensation (Bian et al., this volume). In the worm, the dosage compensation complex (DCC) reduces gene expression by half from the X chromosome in XX hermaphrodites. DCC contains condensin-like subunits that facilitate dosage compensation by forming large ( 1-Mb) TADs. In addition, the silenced $\mathrm{X}$ is enriched for a specific histone mark, H4K20me1. From its crystal structure, Meyer discovered that the DCC subunit DPY-21 belongs to a new Jumonji C demethylase subfamily. DPY-21 converts H4K20me2 to $\mathrm{H} 4 \mathrm{~K} 20 \mathrm{me} 1$ and is required for proper dosage compensation. These demethylases are conserved, providing new tools for studying other functions of H4K20 methylation, such as in DNA replication. This study highlighted the awesome power of structural biology to inform genetics.

Because transcription has been thought to be repressed during mitosis, how are transcriptional programs reestablished to propagate cell fate? Ken Zaret proposed that a low level of transcription is maintained in mitosis, which they detected by ethynyl uridine (EU) labeling (Palozola et al., this volume). Although mitosis disrupts enhancerpromoter interactions, promoters maintain low amplitude transcription. Zaret proposes that this low-level transcription is the long speculated "bookmark" in mitosis that carries the template for the cell's transcriptional program into the next cell cycle. Consistent with earlier talks on loop extrusion and chromosome structure, this work also emphasizes the emerging concept that the organization of mitotic chromosomes is not completely unique, but rather lies on a functional continuum with interphase chromosomes.

In addition to controlling expression of protein-coding genes, epigenetic mechanisms are required to constitutively silence the gene-poor, repetitive regions of genomes packaged into heterochromatin. Although often referred to as "junk DNA," repeats silenced by constitutive heterochromatin have been shown to show both helpful and harmful functions. Antoine Peters and colleagues investigated how Polycomb chromatin modifiers and the DAXX/ATRX chaperone load histone H3.3 and establish constitutive heterochromatin at regions surrounding centromeres during mouse development, a function important for proper chromosome segregation and embryonic development. Yukiko Yamashita explored the function of repetitive satellite DNA in flies, finding that the AATATbinding protein D1 is required for clustering pericentric heterochromatin regions on different chromosomes together into chromocenters (Jagannathan and Yamashita, this volume). D1 mutant cells show increased numbers of micronuclei, leading to the proposal that D1-mediated heterochromatin clustering is necessary to prevent nuclear budding and maintain genome integrity. Aaron Straight discussed how, similar to mechanisms observed in fission yeast, proper constitutive heterochromatin silencing in human cells actually requires some noncoding transcription from these loci. He described that pericentric alpha-satellite DNA repeats, although packaged into heterochromatin, transcribe low levels of RNA. This RNA remains bound to chromatin even during mitosis and directly binds to the H3K9 methyltransferase SUV39H1. This interaction stabilizes SUV39H1's localization at heterochromatin and is necessary to maintain histone methylation and heterochromatic states. Finally, Susan Gasser talked about using C. elegans to study the role of $\mathrm{H} 3 \mathrm{~K} 9$ methylation in preventing DNA damage (Zeller and Gasser, this volume). Surprisingly, worms with complete loss of H3K9 methylation are viable but are temperature-sensitive sterile due to damage-induced germline apoptosis. They find that increased transcription of repetitive elements forms RNA: DNA hybrids, which when unresolved cause replication stress and DNA damage. Future work in this area will likely focus on how repetitive, gene-poor genome regions 
must strike a balance between too much and too little transcription, and the roles of constitutive heterochromatin in preventing DNA damage in a variety of contexts.

\section{CONTROL OF GENOME INTEGRITY BY DNA BREAKS}

We have discussed how chromosomes interface with the chromosome segregation machinery, form higher-order structures, and are regulated epigenetically by histones. These processes depend on the chromosome being continuous. However, DNA can break. DNA breaks can be beneficial, by introducing variation into sexually reproducing populations. These breaks can also be harmful, by causing genome instability or cell death. The fate of DNA breaks depends on the context in which they occur. DNA breaks are not just a local issue for genome stability but also have a global impact on chromosome segregation during meiosis and mitosis.

\section{“Good Cop" Breaks in Meiosis and Germ Cells}

Although DNA breaks can lead to genome damage and mutations, DNA breaks are also essential in meiosis. They facilitate homologous chromosome pairing and crossovers, ensuring both proper chromosome segregation and the shuffling of genetic variation.

Several talks addressed how the location of meiotic DNA breaks is specified. One key protein that controls the locations of meiotic recombination hotspots is PRDM9, a histone methyltransferase with an array of zinc-finger domains. It is one of the fastest evolving proteins in the genome and drives speciation, as different alleles of PRDM9 can be incompatible and cause hybrid sterility in mice. Bernard de Massy described transgenic mice carrying a catalytically inactive PRDM9 variant, which showed that the methyltransferase activity of PRDM9 is essential for H3K4me3 and H3K36me3 deposition and specifying the locations of double-strand breaks (DSBs) in vivo, which in turn supports meiosis progression. How these epigenetic modifications interact with the nucleases that actually introduce DNA breaks is a key issue for future research. Although DSBs are required for meiotic crossovers, far more DSBs occur than crossovers, and how crossovers are controlled is also still a mystery. In $C$. elegans, only one crossover occurs per homolog pair. Monica Colaiácovo reported that these crossovers primarily occur off-center, away from the ends and middle of chromosomes (Saito and Colaiácovo, this volume). The off-center positioning of crossovers results in asymmetric anaphase I bivalent chromosomes. Interestingly, the short and long arms of these bivalents recruit different synaptonemal complex proteins, an asymmetry that appears to be important for the normal timing of cohesion loss between sister chromatids. To test the effect of crossover position, she described an inducible transposon system to target a crossover to specific genomic locations, revealing that a centered crossover leads to premature loss of cohesion. Therefore, asymmetric crossovers and differential protein recruitment to the long and short arms of the resulting bivalents control important aspects of C. elegans meiosis. These findings raise fascinating questions about how chromosome asymmetry is established and sensed.

Additional studies of meiotic crossover control were reported using both in vitro and in vivo approaches. Scott Keeney described progress in recombinant protein purification of the yeast endonuclease Spo11 in complex with its obligate partners Rec102, Rec104, and Ski8, to enable biochemical and structural studies. Structural information will be key to understanding how the Spo11 complex breaks DNA and facilitates homologous recombination (HR). Neil Hunter addressed the mechanisms that trigger the culling of oocytes that fail to successfully complete the resolution of programmed meiotic breaks (Hunter, this volume). Surprisingly, if breaks are not repaired within the normal time frame, a SUMO and HORMA protein signaling system prevents the repair of meiotic DSBs. These unrepaired breaks then trigger apoptosis. Culling of defective oocytes therefore occurs by throwing an ingenious judo-like move; persistent DNA breaks are used against the oocytes that generated them, serving as a memory to survey germline health.

In addition to cell intrinsic properties, germ cell development also requires cues from the tissue microenvironment. Although primordial germ cell (PGC) fate had been thought to be restricted to gametes (i.e., PGCs are thought to be unipotent) and PGC commitment to sexual specialization is thought to be cell autonomous, David Page described work showing that PGCs are actually pluripotent and that sexual specialization is dependent on signals from the genital ridge. A mouse conditional knockout of GATA4 - which obliterates the genital ridge - showed that without the genital ridge, PGCs still migrate but stay pluripotent and do not commit to oogenesis or spermatogenesis. The signaling from the genital ridge and the response in the PGCs is the subject of ongoing studies. The complexity of these germ-soma interactions is interesting, but also makes the precise mechanistic study of sex determination and germ cell differentiation difficult. Towards this end, Mitinori Saitou described using pluripotent stem cells to generate progenitor germ cell-like cells (PGCLCs), which mimic PGCs but do not require co-culturing with gonadal somatic cells (Nagaoka and Saitou, this volume). Using systematic chemical screening, they determined that cyclic AMP signaling plays a pivotal role in PGCLC proliferation. They also find that cell cycle kinetics, transcription profiles, and DNA methylation profiles mimic those of PGCs, making PGCLCs a powerful tool for future study of germ cell fates.

\section{"Bad Cop" Breaks in Mitosis, Somatic Cells, and Cancer}

Whereas the adverse effects of DNA damage are balanced with the essential roles of DNA breaks in meiosis, DNA damage is generally deleterious in mitosis. Several talks described important progress in understanding the 
mechanisms of how DNA damage leads to chromosome missegregation, cell death, or cancer.

DNA damage checkpoints do not catch all errors during DNA replication/S phase, and these uncaught errors then need to be resolved in mitosis or they will cause defects during chromosome segregation. Because of forces from the chromosome segregation machinery, incompletely replicated chromosomal segments, incompletely decatenated DNA or unresolved recombination intermediates in anaphase become DNA structures called ultrafine bridges (UFBs). UFBs are difficult to detect by standard DNA dyes and recruit a specific set of proteins involved in their metabolism. Stephen West described the consequences of persistent UFBs on genome instability (West and Chang, this volume). His group generated a cell line system for studying unresolved Holliday junctions during mitosis (by depleting the nuclease Mus81 and the resolvase GEN1). Importantly, these unresolved Holliday junctions do not appear to trigger the DNA damage response; they persist into mitosis and become UFBs. The UFBs then become single-stranded, a process dependent on the DNA translocase PICH and the helicase BLM. During mitosis, the UFBs break, which normally occurs enzymatically (via Mus81 and GEN1). However, West provided evidence that they can also be broken by mitotic spindle forces. It is estimated that although spindle forces may not be strong enough to break double-stranded DNA, they may be strong enough to break single-stranded DNA. This processing of UFBs is therefore the cell's last chance to resolve joined sister chromatids. However, the process is error-prone, with a high probability of chromosome deletions or chromosome arm-level copy number alterations. A challenge for studying these processes in cells is the short time window for mitosis and the complication that proteins involved in UFB metabolism have functions outside of mitosis. To address this challenge, Ian Hickson is pioneering much needed in vitro study of UFBs (Sarlós et al., this volume). He described single molecule modeling of UFBs using DNA substrates held by multiple optical tweezers. This setup allows visualization of both the identity and the order of binding of UFB proteins, and the optical tweezers allow control of the physical and mechanical properties of DNA. Optical tweezer manipulations revealed that the translocase $\mathrm{PICH}$ has a high affinity for stretched dsDNA, which may be a crucial signal for its recruitment to UFBs.

Elucidating the basic mechanisms of DNA repair is not only of fundamental importance for understanding how cells work, but also has significant implications for cancer therapy. Although DNA metabolism defects lead to genomic instability and promote tumorigenesis, these same defects are also therapeutic vulnerabilities. The poster child for this version of nononcogene addiction is in breast and ovarian cancer, where tumors lacking functional BRCA genes are sensitive to DNA cross-linking agents (such as cisplatin) or to inhibitors of poly ADP-ribose polymerase (PARP). Although PARP inhibitors have been fast-tracked into the clinic, drug resistance is a major barrier to efficacy. Future clinical progress now requires a mechanistic understanding of drug resistance.
Several talks addressed the roles of HR proteins in chemosensitivity and drug resistance. Although BRCA2 is most famous for its established role in HR, recent studies revealed that BRCA2 and its binding partner RAD51 are also important in protecting stressed replication forks from resection and degradation by MRE11. Sharon Cantor described shRNA screens for chemo-resistance in BRCA-deficient tumors (Cantor and Calvo, this volume). She identified chromatin regulators that appear to modulate the replication fork protection function of $\mathrm{BRCA} 2$, supporting the idea that restoration of fork protection can be a chemo-resistance mechanism. However, Maria Jasin cautioned that the essential function of BRCA2 in HR should not be forgotten. In contrast to studies with mouse embryonic stem cells or human tumor cells (Guillemette et al. 2015; Ray Chaudhuri et al. 2016), Jasin showed that it is the HR functions and not fork protection functions that are essential for the viability of nontransformed mammary epithelial cells (Feng and Jasin, this volume). She elucidated a mechanism explaining the lethality of BRCA2 loss involving incomplete DNA replication, UFBs, and cell cycle arrest after mitotic UFB processing. She further showed the important role of BRCA2's HR function in chemoresistance. This work highlights cell type differences in the wiring of essential processes. Daniel Durocher used a comprehensive screening approach to find new genetic vulnerabilities to PARP inhibition and new synthetic lethal interactions with BRCA loss. Surprisingly, cells treated with PARP inhibitors show striking sensitivity to RNaseH2 loss. RNaseH2 corrects the misincorporation of ribonucleotides into DNA; without $\mathrm{RNaseH} 2$, a backup process involving topoisomerase I corrects these lesions instead, and topoisomerase I can leave single-stranded DNA nicks. Inhibited PARP become trapped on this nicked DNA, which appears more cytotoxic than nicks alone. Because the RNase H2 gene is in a locus frequently deleted in certain cancers, PARP inhibition may prove a good therapeutic treatment for those types of cancers.

In summary, organisms maintain a balance between beneficial and detrimental DNA breaks. In germline cells, the evolutionary benefits appear to outweigh the risks, because successful meiosis requires the generation of DNA breaks and crossovers. Nevertheless, extensive damage still requires surveillance, possibly to prevent overexpenditure of energy on inviable offspring. In somatic cells, unintended DNA damage needs to be resolved, and unresolved structures can lead to cell death or the propagation of mutations that may lead to cancer.

\section{NEW METHODS DRIVE NEW DISCOVERY}

Important discoveries can come from the clever application of existing technologies. However, major new progress often originates from new technical approachesmany of which were featured at the meeting. Tom Misteli presented a host of new strategies for high-content deep imaging, including SpotLearn to analyze high-throughput FISH signals and enable large-scale interrogation of 
genome organization at the single-cell level (Gudla et al., this volume). Although chromosome conformation capture methods have provided important information about 3D genome structure, Misteli's experiments show that they miss the true dynamics of chromosome behavior. Martin Hetzer is addressing difficult problems about organelle and tissue aging. He discussed the identification of long-lived proteins and their subcellular localization by combining isotope labeling strategies and serial block-face scanning electron microscopy (SBEM-SEM) in a new technique called nanoSIMS (nano secondary ion mass spec). Iain Cheeseman discussed large-scale genome editing strategies to define what is "essential" about essential genes, and how their functions differ between cell types. Steve Henikoff described CUT\&RUN (Cleavage Under Targets and Release Using Nuclease), a sensitive and powerful alternative to ChIP-based methods for mapping chromatin-associated complexes (Henikoff et al., this volume). CUT\&RUN is already widely adopted. Finally, Aaron Straight described ChAR-seq (chromatin-associated RNA sequencing), a new method for mapping the genomic interactions of all chromatinassociated RNAs genome-wide. These techniques and others will undoubtedly enable discovery of previously unexplored biological phenomena and open doors to new fields of research.

\section{CONCLUSION}

The 82nd Cold Spring Harbor Symposium gave us a vantage point from which to view the future of chromosome biology research. Prior related symposia have swung back and forth in their emphasis on either the description of new phenomena or the elucidation of mechanism, with technological advances usually triggering each swing of the pendulum. The 82 nd symposium caught the pendulum about halfway. A complete description of chromosome structure is coming into focus through genomic approaches, advanced imaging, and mathematical modeling. These techniques have provided a level of information about chromosome organization that, until only recently, was unimaginable. There is certainly more to be learned about chromosome structure genome-wide, but a major task going forward will be to figure out in molecular and mechanistic detail how chromosome substructure forms. The 82 nd symposium highlighted that this effort is well underway with huge progress from biochemical and biophysical studies. By the next symposium, we predict that we will have the same satisfying mechanistic level understanding of motors that generate chromosome loops and link sister chromatids that we have for the motors that organize the mitotic apparatus.

The current state of the chromosome segregation field provides a perspective on the future of the chromosome structure field. First, in part through genetic screens, we know most of the players that enable proper chromosome segregation. The field is now focusing on the assembly principles and how intermediate level structures-for example, spindle midzones and the PCM - are assembled from these parts. Similar assembly principles are now also being used for chromosome structure to describe how loop extrusion motors form intermediate structures like chromosome loops. Second, mathematical modeling of spindle microtubule dynamics and interactions has enabled the formulation of quantitative models with testable predictions. The spectacular success of the quantitative iteration of the loop extrusion model shows that this era has already arrived for chromosome structure. Third, as work on the phase transition of pericentriolar proteins illustrated, studying the material properties of biopolymers can identify new functions that emerge from protein ensembles. This kind of work is in its infancy for chromosome biology, but similar work on the synaptonemal complex, for example, might lead to insight about how the numbers and position of crossovers are regulated in meiosis. Finally, how asymmetry is generated during cell division has long been a focus in the cell polarity and chromosome segregation fields. By the next symposia, we hope to have this level of understanding for chromosome structure problems such as the asymmetric distribution of histones and the asymmetric organization of $C$. elegans bivalents reported at the 82nd Symposium.

Although the current symposium highlighted great progress in defining chromosome structure, much remains to be understood about its functional consequences. For example, it is clear that cohesin proteins are required to form TADs, and that genome editing of TAD boundaries has established effects on transcription. Therefore, the role of mutated cohesin subunits in tumorigenesis and cancer has been attributed to misregulated transcription. However, transcriptional profiling of cells after cohesin inactivation shows surprisingly subtle effects, with only small-scale changes affecting many genes (Rao et al. 2017). Either tumorigenesis results from alterations in a small number of exquisitely dosage sensitive genes or, probably more likely, it emerges from the aggregate alteration of many genes. Understanding the combinatorial effects of many small changes is a challenging general problem in biology. Tackling this in the future will require new methods; perhaps analytic approaches used to study polygenic traits will be relevant (Boyle et al. 2017). Also, in addition to transcription, perhaps by the next meeting it will be apparent that many other long-range chromosome interaction phenomena, such as VDJ recombination, require loop extrusion.

The chromosome segregation field has long focused on microtubules and their assembly into spindles, but the field is now bringing the nucleus back into focus. The coordination between chromosome segregation and the nuclear envelope is still being elucidated in metazoan cells. We have known for 100 years that mitotic defects can lead to aneuploidy; however, we have only recently fully appreciated the impact mitotic errors have on the nuclear envelope and the structural integrity of chromosomes. For example, mitotic errors can lead to chromosome breakage at common fragile sites, as well as the mutational process termed chromothripsis. Mitotic errors can therefore drive rapid genome changes and evolution. This has obvious relevance to cancer. By the next symposium, we will have a much better, perhaps comprehensive, 
understanding of abnormal nuclear architecture in cancer and the mutational processes that drive cancer genome evolution. These insights will hopefully lead to new ideas about therapy; for example, recent work has revealed unexpected links between nuclear envelope integrity and innate immune signaling that may, in some contexts, serve as a unique cancer vulnerability (Harding et al. 2017; Mackenzie et al. 2017; Bakhoum et al. 2018).

Although much has been learned from model systems like HeLa cells, it is now clear that there is a host of important unsolved chromosome segregation problems that must be studied in their natural habitat. As mentioned by Iain Cheeseman, cell type-specific regulation of chromosome segregation is clearly important, but relatively understudied. We also need to understand how the 3D architecture of tissues impacts chromosome segregationhopefully in quantitative terms. Fully understanding how cell biological processes impact organism-level processes such as development or disease will require the combination of in vivo models with new advances in cellular imaging.

Summing up a meeting as rich and diverse as the 82nd Cold Spring Harbor Symposium is a dangerous business. The temptation is to shoehorn the presentations into a few predefined themes. We have tried to highlight important themes in this summary, but we also do not want to ignore the pleasing scientific diversity. As Isaiah Berlin pointed out in his famous essay The Hedgehog and the Fox, many writers and thinkers want to be "hedgehogs" with one big idea (e.g., Plato, Hegel, Dostoevsky). However, it is the "foxes" (e.g., Shakespeare, Goethe, Balzac) that appreciate the plurality of human types and experiences, and who may in fact get closer to the truth. Looking over past meetings, one is struck by the frequency with which unexpected findings, often in obscure model systems, led to creative work that changed the direction of the whole field. In his Dorcas Cummings Lecture, David Page not only took us on an entertaining tour of the checkered evolutionary history of the human Y chromosome but also threw a pitch from way out in left field. He proposed that by ignoring the possibility of sex-specific cell autonomous differences, we may be missing fundamental information about cells and chromosomes. Although we cannot predict with certainty which "out-there" ideas from the 82 nd symposium will become a dominant theme in the next chromosome segregation and structure symposium, we will all be back to look for them.

\section{ACKNOWLEDGMENTS}

We would like to thank the National Institutes of Health (5R01 CA213404-20) and the Howard Hughes Medical Institute for support.

\section{REFERENCES}

Alipour E, Marko JF. 2012. Self-organization of domain structures by DNA-loop-extruding enzymes. Nucleic Acids Res 40: $11202-11212$.
Bakhoum SF, Ngo B, Laughney AM, Cavallo J-A, Murphy CJ, Ly P, Shah P, Sriram RK, Watkins TBK, Taunk NK, et al. 2018. Chromosomal instability drives metastasis through a cytosolic DNA response. Nature 553: 467-472.

Boyle EA, Li YI, Pritchard JK. 2017. An expanded view of complex traits: From polygenic to omnigenic. Cell 169: 11771186.

Earnshaw WC, Bernat RL. 1991. Chromosomal passengers: Toward an integrated view of mitosis. Chromosoma 100: 139-146.

Fussner E, Strauss M, Djuric U, Li R, Ahmed K, Hart M, Ellis J, Bazett-Jones DP. 2012. Open and closed domains in the mouse genome are configured as 10-nm chromatin fibres. EMBO Rep 13: 992-996.

Ganji M, Shaltiel IA, Bisht S, Kim E, Kalichava A, Haering CH, Dekker C. 2018. Real-time imaging of DNA loop extrusion by condensin. Science 360: 102-105.

Gibcus JH, Samejima K, Goloborodko A, Samejima I, Naumova N, Nuebler J, Kanemaki MT, Xie L, Paulson JR, Earnshaw WC, et al. 2018. A pathway for mitotic chromosome formation. Science 359: eaao6135.

Giorgetti L, Lajoie BR, Carter AC, Attia M, Zhan Y, Xu J, Chen CJ, Kaplan N, Chang HY, Heard E, et al. 2016. Structural organization of the inactive $\mathrm{X}$ chromosome in the mouse. Nature 535: 575-579.

Guillemette S, Serra RW, Peng M, Hayes JA, Konstantinopoulos PA, Green MR, Cantor SB. 2015. Resistance to therapy in BRCA2 mutant cells due to loss of the nucleosome remodeling factor CHD4. Genes Dev 29: 489-494.

Haarhuis JHI, van der Weide RH, Blomen VA, Yáñez-Cuna JO, Amendola M, van Ruiten MS, Krijger PHL, Teunissen H, Medema RH, Van Steensel B, et al. 2017. The cohesin release factor WAPL restricts chromatin loop extension. Cell 169: 693-700.e14.

Harding SM, Benci JL, Irianto J, Discher DE, Minn AJ, Greenberg RA. 2017. Mitotic progression following DNA damage enables pattern recognition within micronuclei. Nature 548: 466-470.

Hatch EM, Fischer AH, Deerinck TJ, Hetzer MW. 2013. Catastrophic nuclear envelope collapse in cancer cell micronuclei. Cell 154: 47-60.

Henikoff S, Malik HS. 2002. Centromeres: Selfish drivers. Nature 417: 227.

Henikoff S, Ahmad K, Malik HS. 2001. The centromere paradox: Stable inheritance with rapidly evolving DNA. Science 293: 1098-1102.

Lieberman-Aiden E, van Berkum NL, Williams L, Imakaev M, Ragoczy T, Telling A, Amit I, Lajoie BR, Sabo PJ, Dorschner $\mathrm{MO}$, et al. 2009. Comprehensive mapping of long-range interactions reveals folding principles of the human genome. Science 326: 289-293.

Mackenzie KJ, Carroll P, Martin C-A, Murina O, Fluteau A, Simpson DJ, Olova N, Sutcliffe H, Rainger JK, Leitch A, et al. 2017. cGAS surveillance of micronuclei links genome instability to innate immunity. Nature 548: 461-465.

Mazia D. 1961. Mitosis and the physiology of cell division. Elsevier, New York.

Nasmyth K. 2001. Disseminating the genome: Joining, resolving, and separating sister chromatids during mitosis and meiosis. Annu Rev Genet 35: 673-745.

Nishino Y, Eltsov M, Joti Y, Ito K, Takata H, Takahashi Y, Hihara S, Frangakis AS, Imamoto N, Ishikawa T, et al. 2012. Human mitotic chromosomes consist predominantly of irregularly folded nucleosome fibres without a $30-\mathrm{nm}$ chromatin structure. $E M B O J$ 31: 1644-1653.

Rao SSP, Huang S-C, Hilaire BGS, Engreitz JM, Perez EM, Kieffer-Kwon K-R, Sanborn AL, Johnstone SE, Bascom GD, Bochkov ID, et al. 2017. Cohesin loss eliminates all loop domains. Cell 171: 305-309.e24.

Ray Chaudhuri A, Callen E, Ding X, Gogola E, Duarte AA, Lee J-E, Wong N, Lafarga V, Calvo JA, Panzarino NJ, et al. 2016. Replication fork stability confers chemoresistance in BRCAdeficient cells. Nature 535: 382-387. 
Riggs AD. 1990. DNA methylation and late replication probably aid cell memory, and type I DNA reeling could aid chromosome folding and enhancer function. Philos Trans R Soc Lond B Biol Sci 326: 285-297.

Subramanian R, Ti S-C, Tan L, Darst SA, Kapoor TM. 2013. Marking and measuring single microtubules by PRC1 and kinesin-4. Cell 154: 377-390.

Tedeschi A, Wutz G, Huet S, Jaritz M, Wuensche A, Schirghuber E, Davidson IF, Tang W, Cisneros DA, Bhaskara V, et al. 2013. Wapl is an essential regulator of chromatin structure and chromosome segregation. Nature 501: 564-568.
Terakawa T, Bisht S, Eeftens JM, Dekker C, Haering CH, Greene EC. 2017. The condensin complex is a mechanochemical motor that translocates along DNA. Science 358: 672-676.

Wang X, Brandão HB, Le TBK, Laub MT, Rudner DZ. 2017. Bacillus subtilis SMC complexes juxtapose chromosome arms as they travel from origin to terminus. Science 355: 524-527.

Wutz G, Várnai C, Nagasaka K, Cisneros DA, Stocsits RR, Tang W, Schoenfelder S, Jessberger G, Muhar M, Hossain MJ, et al. 2017. Topologically associating domains and chromatin loops depend on cohesin and are regulated by CTCF, WAPL, and PDS5 proteins. EMBO J 36: 3573-3599. 


\section{$\$_{\text {CSH }}^{\infty}$ Cold Spring Harbor Symposia SYMPOSIA on Quantitative Biology}

\section{How the Genome Folds, Divides, Lives, and Dies}

Whitney L. Johnson, Kathleen T. Xie, Mijung Kwon, et al.

Cold Spring Harb Symp Quant Biol 2017 82: 349-360 originally published online May 25, 2018 Access the most recent version at doi:10.1101/sqb.2017.82.035527

References This article cites 26 articles, 10 of which can be accessed free at: http://symposium.cshlp.org/content/82/349.full.html\#ref-list-1
Creative This article is distributed under the terms of the
Commons http://creativecommons.org/licenses/by-nc/4.0/, which permits reuse and License redistribution, except for commercial purposes, provided that the original author and source are credited.

Email Alerting
Service $\quad \begin{gathered}\text { Receive free email alerts when new articles cite this article - sign up in } \\ \text { the box at the top right corner of the article or click here. }\end{gathered}$ 